\title{
A Leishmania amazonensis ZIP family iron transporter is essential for parasite replication within macrophage phagolysosomes
}

\author{
Chau Huynh, ${ }^{1}$ David L. Sacks, ${ }^{3}$ and Norma W. Andrews ${ }^{1,2}$
}

'Section of Microbial Pathogenesis and ${ }^{2}$ Department of Cell Biology, Yale University School of Medicine, New Haven, CT 06510 ${ }^{3}$ Laboratory of Parasitic Diseases, National Institutes of Allergy and Infectious Diseases, National Institutes of Health, Bethesda, MD 20892

Infection of mammalian hosts with Leishmania amazonensis depends on the remarkable ability of these parasites to replicate within macrophage phagolysosomes. A critical adaptation for survival in this harsh environment is an efficient mechanism for gaining access to iron. In this study, we identify and characterize LIT1, a novel L. amazonensis membrane protein with extensive similarity to IRT1, a ZIP family ferrous iron transporter from Arabidopsis thaliana. The ability of LIT1 to promote iron transport was demonstrated after expression in yeast and in L. amazonensis LIT1-null amastigotes. Endogenous LIT1 was only detectable in amastigotes replicating intracellularly, and its intracellular expression was accelerated under conditions predicted to result in iron deprivation. Although L. amazonensis lacking LIT1 grew normally in axenic culture and had no defects differentiating into infective forms, replication within macrophages was abolished. Consistent with an essential role for LIT1 in intracellular growth as amastigotes, $\Delta$ lit1 parasites were avirulent. After inoculation into highly susceptible mice, no lesions were detected, even after extensive periods of time. Despite the absence of pathology, viable $\Delta$ lit 1 parasites were recovered from the original sites of inoculation, indicating that $L$. amazonensis can persist in vivo independently of the ability to grow in macrophages. Our findings highlight the essential role played by intracellular iron acquisition in Leishmania virulence and identify this pathway as a promising target for therapeutic intervention.

\section{CORRESPONDENCE}

Norma W. Andrews:

norma.andrews@yale.edu

Abbreviations used: BMmø, bone marrow-derived macrophage(s); BPS, bathophenanthroline disulfonic acid; DLN, draining lymph node; ORF, open reading frame.
Limiting the access of pathogens to iron is thought to be one of the defense strategies used by activated macrophages against intracellular infections (1). The critical role of iron for the survival of microbes within endocytic vesicles is highlighted by the importance of Nramp1 (also known as Slc11a1) in natural resistance to infections (2). Point mutations in Nramp1 are associated with the susceptibility of macrophages to pathogens that replicate within the endocytic compartment, such as the bacteria Salmonella and Mycobacterium and the protozoan Leishmania. Recent investigations into the role of Nramp1 revealed that it functions as a $\mathrm{pH}$-dependent transporter that can extrude $\mathrm{Mn}^{2+}$, and possibly other divalent cations, from phagolysosomes (3). Thus, although this has not yet been directly demonstrated, Nramp1 may promote resistance to infection by removing iron from intracellular compartments, starving pathogens

The online version of this article contains supplemental material of this essential micronutrient (4). Because several additional mechanisms contribute to the limited supply of iron that is intracellularly available, an efficient iron uptake mechanism is critical for the survival and replication of intracellular pathogens.

Iron is critically required as a cofactor of various important enzymes. In Leishmania, which replicates inside phagolysosomes of macrophages, iron superoxide dismutases are important for protection against the oxidative damage resulting from activation of the NADPH oxidase (5). The uptake of iron for these essential enzymatic functions poses a particular problem for both pathogens and host cells (6). Ferrous iron $\left(\mathrm{Fe}^{2+}\right)$ is soluble in biological fluids, but it cannot be allowed to accumulate because of the highly toxic hydroxyl radicals that it generates by the Fenton reaction in the presence of oxygen. Therefore, iron uptake and storage have to be carefully regulated in both prokaryotes and eukaryotes (6-8). In an aerobic environment at 
neutral $\mathrm{pH}$, iron exists as the poorly soluble oxidized ferric $\left(\mathrm{Fe}^{3+}\right)$ form, which is found complexed to transferrin in serum, to lactoferrin in mucosal secretions, and to ferritin in the cytosol. Mammalian cells internalize a complex of transferrin with two $\mathrm{Fe}^{3+}$ ions (holotransferrin), which is endocytosed after binding to the transferrin receptor. Inside endosomes, acidification causes $\mathrm{Fe}^{3+}$ to be released from transferrin, followed by reduction to $\mathrm{Fe}^{2+}$ (9) and translocation to the cytosol (10). Thus, the low amounts of iron that are allowed to accumulate inside late endosomes and lysosomes are mostly in the insoluble
$\mathrm{Fe}^{3+}$ form, creating a particular challenge for pathogens residing in these compartments.

Many bacteria internalize iron complexed to siderophores, secreted molecules that effectively compete with host proteins for $\mathrm{Fe}^{3+}$ (11). There is so far no evidence that protozoa can acquire iron through siderophores and siderophore receptors; a specific investigation of this issue revealed that secreted Leishmania chagasi molecules are not capable of removing $\mathrm{Fe}^{3+}$ from lactoferrin or transferrin (12). On the other hand, receptor-mediated transferrin uptake has been

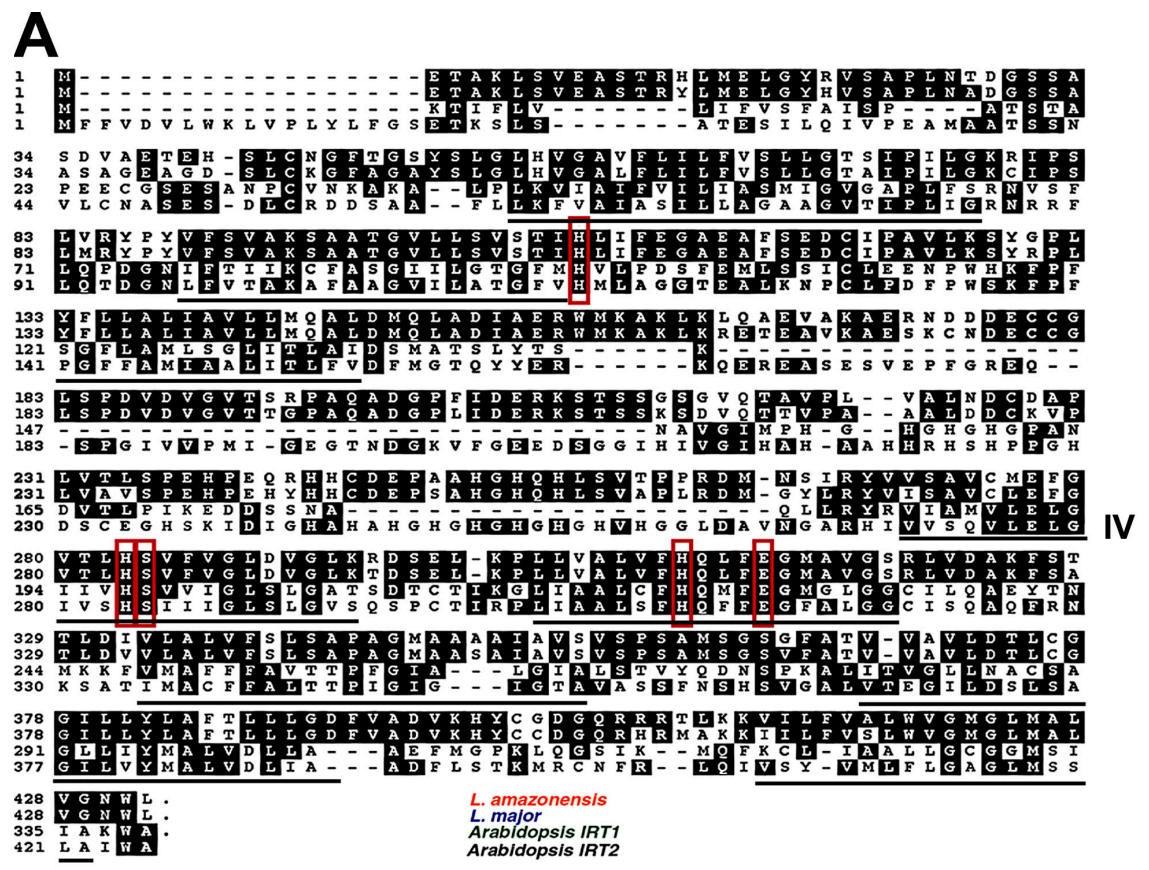

B

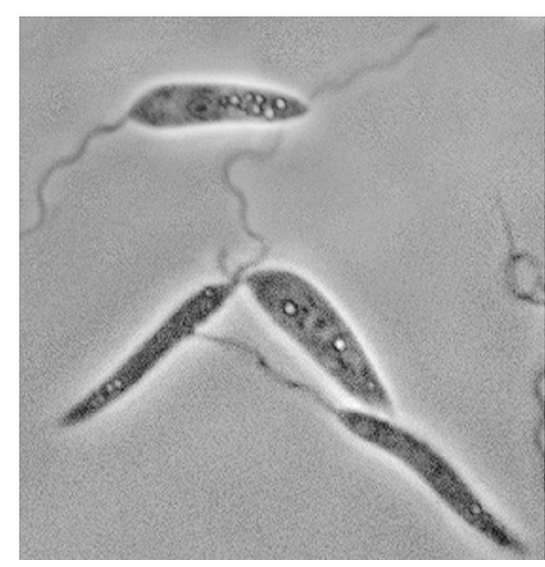

Figure 1. The Leishmania LIT1 gene encodes a membrane protein with high similarity to the IRT1 ferrous iron transporter from $A$. thaliana. (A) Alignment of the amino acid sequences of LIT1 from L. amazonensis and L. major (LmjF31.3060 and LmjF31.3070), and the IRT1 and IRT2 genes from $A$. thaliana. Identical residues are boxed in black, and the conserved residues shown to be critical for iron transport function in

\section{GFP-LIT1}

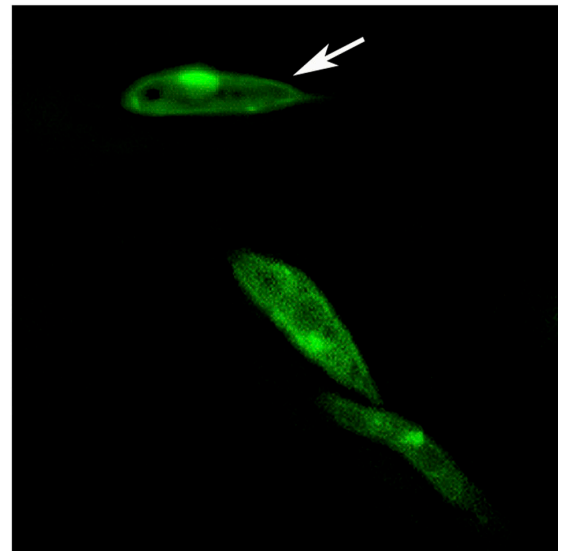

IRT1 are outlined in red. The predicted transmembrane domains (I-VIII) are underlined, and transmembrane domain IV is indicated. (B) Wild-type L. amazonensis promastigotes transfected with a GFP-LIT1 expression construct (pXG-LIT1). GFP-LIT1 fluorescence was detected on a plasma membrane (arrow) and in an additional intracellular, perinuclear compartment likely to correspond to the parasite's megasome. 
extensively characterized in the trypanosomatid parasite Trypanosoma brucei (13). Although Leishmania also appears capable of acquiring $\mathrm{Fe}^{3+}$ complexed with lactoferrin or transferrin $(12,14)$, the existence of a specific receptor-based uptake mechanism in these parasites has not been confirmed (6). An important development in this area came from the recent observation that iron uptake in $L$. chagasi occurs preferentially in the reduced $\mathrm{Fe}^{2+}$ form. This finding led to the demonstration that Leishmania contains an NADPH-dependent iron reductase activity capable of converting $\mathrm{Fe}^{3+}$ into the more soluble ferrous $\mathrm{Fe}^{2+}(15)$. Thus, instead of relying on a transferrin receptor-based mechanism to take up the chelated ferric iron that is available intracellularly, it appears that Leishmania can generate soluble ferrous iron for direct uptake through membrane transporters. In this study we characterize LIT1, the first ferrous iron transporter to be identified in Leishmania. Our findings demonstrate that LIT1 is essential for replication of the parasites within macrophage phagolysosomes and for the development of pathology in mice.

\section{RESULTS}

The Leishmania genome encodes two copies of LIT1, a homologue of the IRT1 iron transporter-encoding gene of Arabidopsis thaliana

Homology searches of the L. major database identified two identical genes on chromosome 31 in tandem, LmjF31.3060 and LmjF31.3070 (designated LIT1-1 and LIT1-2; available from GenBank/EMBL/DDBJ under accession no. CT005268; Fig. 1 A), which share 30\% identity and $53.8 \%$ similarity with IRT1 from $A$. thaliana (available from GenBank/EMBL/ DDBJ under accession no. U27590). PCR amplification and cloning of the corresponding 1.3-kb LIT1 gene from genomic DNA of $L$. amazonensis revealed extensive identity with the L. major sequence (Fig. 1 A).

Arabidopsis IRT1 is an $\mathrm{Fe}^{2+}$ transporter from the ZIP family, whose members range from 309 to 476 amino acids and are predicted to have a similar membrane topology, with eight transmembrane domains and the amino- and carboxyterminal ends located on the extracellular side of the plasma membrane (16). Completely consistent with these features, LIT1 encodes a 432 -amino acid protein of $50 \mathrm{kD}$, which is also predicted to contain eight transmembrane domains. The most conserved portion of proteins from the ZIP family is in the putative transmembrane domain IV. This region is predicted to form an amphipathic helix with a conserved histidine and an adjacent semipolar residue, which are thought to be essential components of the heavy metal binding site $(16,17)$. Extensive identity is observed throughout this region in two homologous Arabidopsis genes, IRT1 and IRT2 (available from GenBank/EMBL/DDBJ under accession no. NM_118088), and the LIT1 genes from both L. major and L. amazonensis (Fig. 1 A). Furthermore, there is complete conservation of all five residues shown to be essential for divalent metal transport by Arabidopsis IRT1 (Fig. 1 A, residues boxed in red) (17), reinforcing the possibility that the Leishmania LIT1 genes encode an iron transporter. When

expressed in L. amazonensis promastigotes, a GFP-tagged form of LIT1 was expressed in a pattern consistent with localization on the plasma membrane (Fig. 1 B). The GFPLIT1 chimera was also detected in an intracellular compartment, which may correspond to the parasite's megasome, a lysosome-like compartment $(18,19)$.

\section{A \\ $1 \mathrm{~h}$ \\ $24 \mathrm{~h}$}
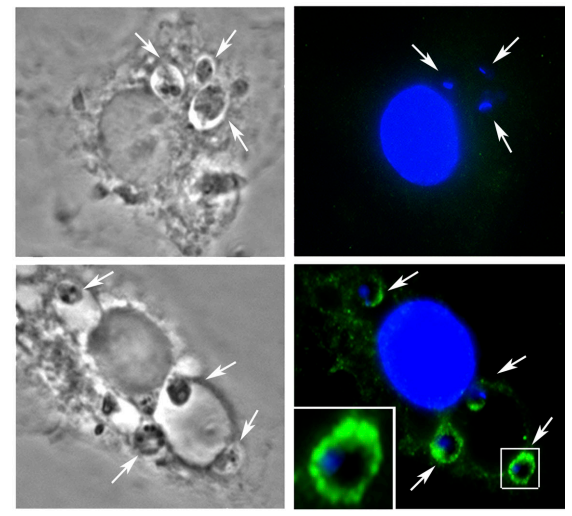

B
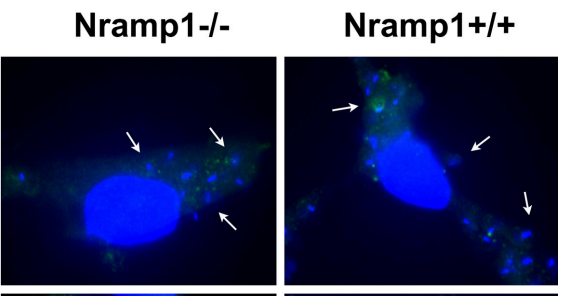

$6 h$
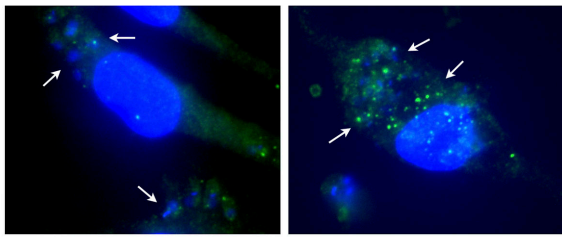

$12 \mathrm{~h}$
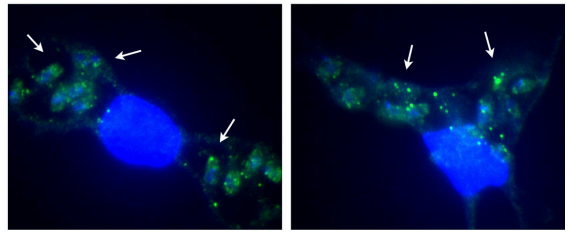

Figure 2. L. amazonensis LIT1 is expressed by amastigotes residing intracellularly, and expression is accelerated in Nramp1+/+ macrophages. Mouse BMmø were infected with L. amazonensis axenic amastigotes for $1 \mathrm{~h}$, washed, and further incubated for the indicated periods of time, followed by fixation, permeabilization, and immunofluorescence with antibodies specific for LIT1. (A) C57BL/6 BMmø (Nramp1-1-). Phase contrast (left) and fluorescence microscopy (right) images are shown. LIT1 was only detected $24 \mathrm{~h}$ after infection in a pattern consistent with localization on the plasma membrane of amastigotes (the inset shows an enlarged image). (B) C57BL/10ScSn (Nramp1 ${ }^{-1-}$ ) or B10.L-Lsh (Nramp1 ${ }^{+/+}$) BMmø. Punctate LIT1 immunofluorescence was detected after $12 \mathrm{~h}$ in the Nramp $1^{-1-}$ BMmø and after $6 \mathrm{~h}$ in the Nramp1 ${ }^{-1-}$ BMm $\varnothing$. Antibodies to LIT1 are labeled in green, and the host cell and parasite's DNA are stained in blue (DAPI). Arrows in A and B point to intracellular parasites. The images were acquired and enhanced for contrast under identical conditions. 
LIT1 is expressed by intracellular amastigotes and functions as a divalent metal transporter with preference for iron Antibodies generated against the 15 amino-terminal amino acids of LIT1, a region of the protein that is predicted to be exposed extracellularly (16), failed to detect endogenous LIT1 by immunofluorescence or Western blot in L. amazonensis promastigotes and axenically grown amastigotes (not depicted). However, when immunofluorescence was performed on permeabilized bone marrow-derived macrophages (BMmø) from C57BL/6 mice infected with axenic amastigotes, a strong reaction with the antibodies was observed on parasites residing intracellularly for $24 \mathrm{~h}$. This is a stage when the markedly enlarged parasitophorous vacuoles typical of L. amazonensis infections are clearly visible (Fig. 2 A, bottom). LIT1 was detected around the periphery of intracellular amastigotes, which was consistent with its predicted plasma membrane localization (Fig. $2 \mathrm{~A}$, bottom right, inset).

Consistent with what was observed in axenically grown promastigotes and amastigotes, LIT1 was not detected by immunofluorescence on amastigotes recently internalized in BMmø (Fig. 2 A, top). This finding strongly suggested that LIT1 expression is up-regulated in the intracellular environment. Expression of IRT1, the close homologue of LIT1 in $A$. thaliana, is induced under iron-deficient conditions (20). Because Nramp1/Slc11a1 has been postulated to modulate the iron concentration of phagolysosomes (4), we performed time-course infection experiments in BMmø from C57BL/ 10ScSn $\left(\mathrm{Nramp}^{-/-}\right)$or B10.L-Lsh $\left(\mathrm{Nramp} 1^{+/+}\right)$congenic mice and analyzed LIT1 expression by immunofluorescence. In the Nramp1 $1^{-/-}$BMmø, LIT1 expression was low at $6 \mathrm{~h}$ after infection but was clearly detected in a punctate pattern after 12 h (Fig. 2 B, Nramp ${ }^{-/}$). In Nramp1 ${ }^{+/+}$BMmø, LIT1 expression was detected earlier; punctate immunofluorescence associated with amastigotes was clearly visible at $6 \mathrm{~h}$ after infection (Fig. $2 \mathrm{~B}, \mathrm{Nramp}^{+/+}$). Confirming what was seen in C57BL/6 BMmø (Nramp1 $1^{-1-}$ ), no LIT1-specific immunofluorescence was detected in BMmø derived from both mouse strains immediately after the 1-h infection period (Fig. 2 B, top). These results (see also Fig. S1, available at http://www.jem.org/cgi/content/full/jem.20060559/DC1) suggest that the intracellular expression of LIT1 may be accelerated by the Nramp1-mediated extrusion of iron from the Leishmania-containing phagolysosome.

To confirm that L. amazonensis LIT1 functions as an iron transporter, we performed assays of functional complementation in yeast. The Sacharomyces cerevisiae $\Delta$ fet 3 fet 4 mutant strain is extremely sensitive to iron deprivation, because it lacks the FET3 multicopper oxidase required for high affinity $\mathrm{Fe}^{2+}$ transport and the FET4 low affinity $\mathrm{Fe}^{2+}$ transporter (21). It grows in iron-rich medium (YPD) but not in medium containing the iron chelator bathophenanthroline disulfonic acid (BPS; Fig. 3 A). The Arabidopsis IRT1 gene was originally identified in a functional complementation screen using this strain and was subsequently shown to encode an iron transporter with preference for $\mathrm{Fe}^{2+}$ as a substrate (22). Overexpression of L. amazonensis LIT1 using the yeast multicopy vector p426 (23) suppressed the $\Delta$ fet3fet 4 growth defect in iron-limited conditions (YPD $+20 \mu \mathrm{M}$ BPS; Fig. 3 A), directly demonstrating that $\mathrm{Fe}^{2+}$ transport ability was restored. To examine the divalent metal substrate preference of LIT1, a wild-type $S$. cerevisiae strain was transformed with LIT1 and grown in minimum medium (SCD) containing cadmium, which is toxic for yeast at high concentrations (24). LIT1 overexpression increased the sensitivity of $S$. cerevisiae to low concentrations of cadmium, but the growth defect was suppressed when cadmium and iron were provided simultaneously (Fig. 3 B). This result demonstrates, similar to what was previously shown for the Arabidopsis IRT1 transporter (17), that though LIT1 is capable of translocating cadmium, it has a preference for iron as a substrate.

\section{LIT1 is not required for differentiation and growth of $L$. amazonensis in axenic culture}

The two identical LIT1 genes are present in tandem within a 5,425 -bp region, allowing us to generate a targeted deletion construct to simultaneously inactivate both copies (Fig. 4 A). Because Leishmania species are diploid protozoan parasites, two rounds of gene disruption are required to generate null clones. After two rounds of transfection and selection, clonal lines were identified in which both alleles of the two LIT1 copies had been replaced by insertion of the Hyg- and

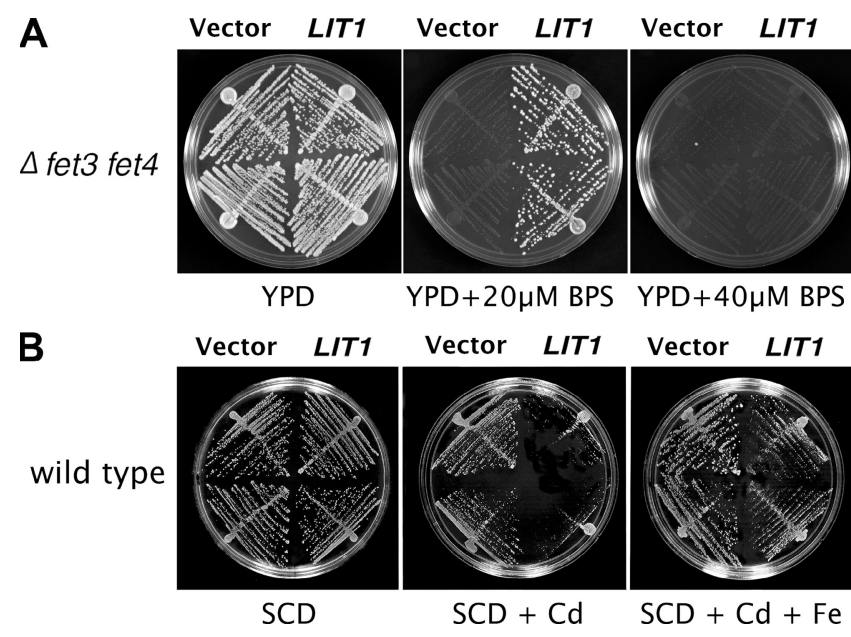

Figure 3. L. amazonensis LIT1 functions as a divalent metal transporter with preference for iron. (A) LIT1 functionally replaces iron transporters in S. cerevisiae. The iron transport-deficient $\Delta$ fet3fet4 strain, carrying either empty vector or L. amazonensis LIT1, was streaked on YPDagar or YPD-agar containing 20 or $40 \mu \mathrm{M}$ of the iron chelator BPS. Both strains, vector or LIT1 transformed, were able to grow in iron-rich conditions (YPD). However, only the strain transformed with LIT1 was able to grow under limiting iron conditions (YPD $+20 \mu \mathrm{M}$ BPS). Extreme iron chelation conditions (YPD $+40 \mu \mathrm{M}$ BPS) prevented the growth of both strains, vector or LIT1-transformed. (B) LIT1 has preference for iron over cadmium as a substrate. Overexpression of LIT1 in wild-type S. cerevisiae caused lethality when grown in the presence of $100 \mu \mathrm{M} \mathrm{CdCl}$ (SCD $+C d$ ). The lethality caused by enhanced transport of $\mathrm{Cd}$ into the cells was overcome by supplying $\mathrm{FeCl}_{3}$ as an additional substrate ( $\left.\mathrm{SCD}+\mathrm{Cd}+\mathrm{Fe}\right)$. 
A

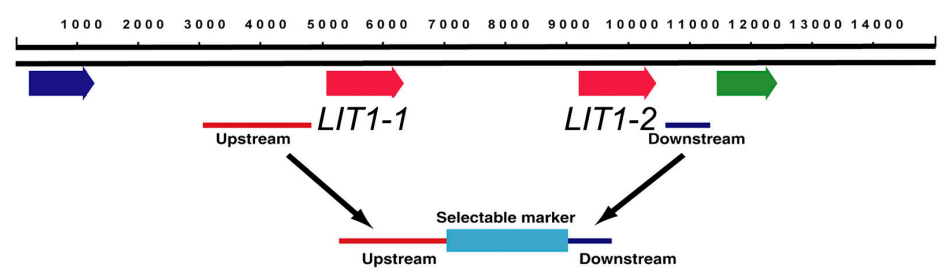

Targeted gene deletion
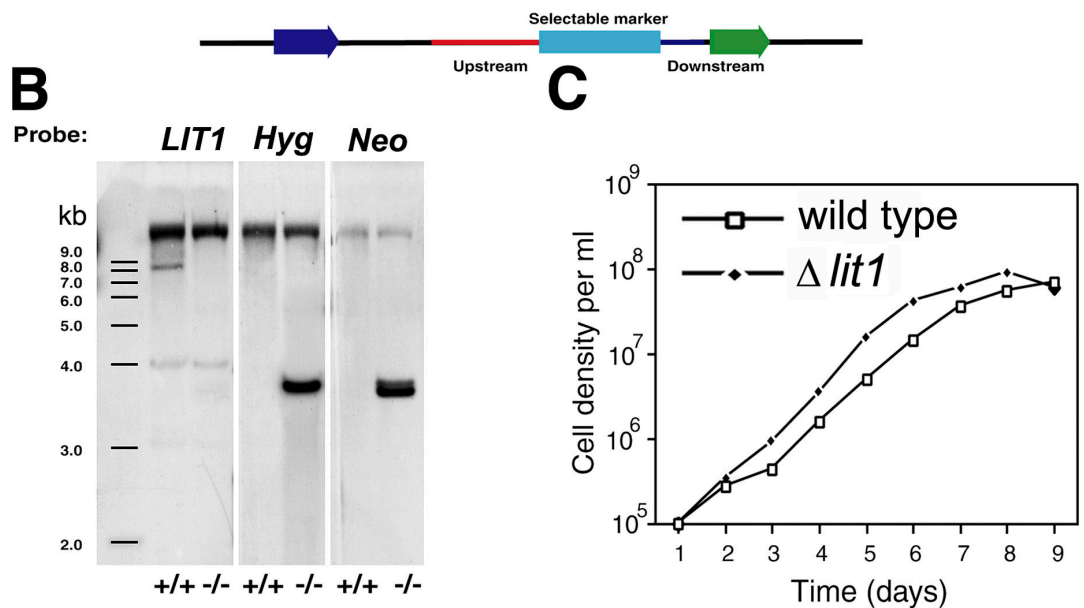

D

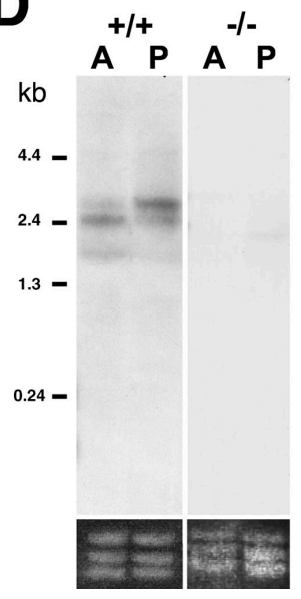

E
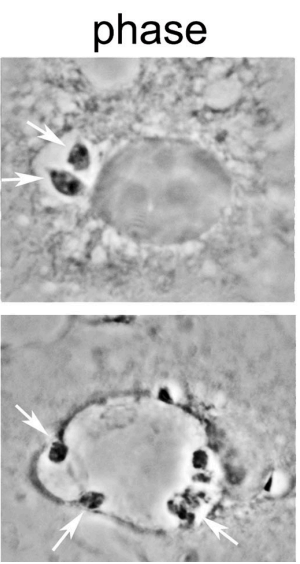

DAPI
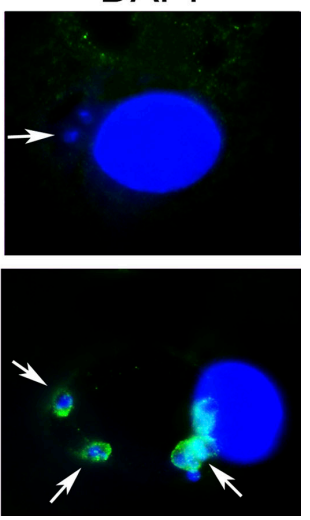

Figure 4. LIT1-null L. amazonensis grow normally as promastigotes and generate infective stages capable of infecting macrophages. Generation and characterization of LIT1-null mutants.

(A) Generation of $\Delta$ lit1 mutants. The diagram shows the two LIT1 genes in tandem (LIT1-1 and LIT11-2; red arrows), flanked by two ORFs of unknown function (blue and green arrows). Upstream and downstream sequences were targeted for replacing the LIT1 genes with selectable markers (light blue), without disruption of upstream and downstream genes. (B) Southern blots of genomic DNA from wild-type $(+\mid+)$ or homozygous $\Delta$ lit1 $(-1-)$ promastigotes digested with Notl and probed with full-length LIT1, Hyg, or Neo ORFs. The successful sequential replacement of both alleles of LIT1-1 and LIT1-2 is indicated by the LIT1-containing 8-kb fragment only in wild-type parasites, and by both Hyg-and Neo-containing less than 4-kb fragments only in the null parasites. (C) Growth curves of wild-type and $\Delta$ lit 1 promastigotes in liquid culture. No differences in growth were detected, and $\Delta$ lit1 stationary phase promastigotes differentiated normally into infective metacyclic forms. (D) Northern blot of total RNA from wild-type $(+/+)$ and $\Delta$ lit $(-/-)$ axenic promastigotes $(\mathrm{P})$ and amastigotes (A) probed with full-length LIT1. rRNA loading controls are shown at the bottom. (E) Infection of BMmø by wild-type and $\Delta$ lit1 axenic amastigotes. $\Delta /$ lit 1 promastigotes differentiated normally into infective axenic amastigotes, infected macrophages, and induced initial expansion of the parasitophorous vacuole. LIT1 was detected by immunofluorescence on wild-type intracellular amastigotes $24 \mathrm{~h}$ after infection but not on $\Delta$ lit1 amastigotes. Anti-LIT1 antibodies are labeled in green, and host cell and parasite DNA are labeled in blue (DAPI). Arrows point to intracellular amastigotes. 
Neo-selectable markers (Fig. 4 B). Independent null clones isolated in this manner behaved similarly, so only results with a single doubly disrupted clonal line, designated $\Delta$ lit1, are shown. Null $\Delta$ lit1 promastigotes had no growth defect in culture as promastigotes (Fig. $4 \mathrm{C}$ ) and generated comparable numbers of infective metacyclic promastigotes when the cultures reached stationary phase (slender, free-swimming metacyclics were counted after agglutination of procyclic promastigotes with the mAb 3A1; unpublished data) (25). Similarly, no defects in differentiation and growth as axenic amastigotes were observed after the cultures were shifted to $\mathrm{pH} 5.5$ and incubated at $32^{\circ} \mathrm{C}$ (unpublished data). Northern blots identified a transcript of $\sim 2.4-2.5 \mathrm{~kb}$ in wild-type parasites but not in $\Delta$ lit1 axenic amastigotes and promastigotes (Fig. 4 D). The LIT1 protein was only detected with antibodies in intracellular amastigotes (as described in the previous section; Fig. 2) or in extracellular promastigotes and amastigotes overexpressing LIT1 (see the next section). These observations reinforce the possibility that LIT1 expression is regulated posttranslationally in response to iron deprivation, as previously shown for several transitional metal transporters $(20,26,27)$.

\section{LIT1 promotes iron transport and is required} for intracellular replication in macrophages

The normal generation of infective axenic amastigotes from $\Delta$ lit1 promastigotes allowed us to perform macrophage infections in parallel with wild-type parasites. The numbers of intracellular parasites detected after $1 \mathrm{~h}$ of infection were similar in BMmø infected with the wild-type or $\Delta$ lit1 lines, indicating no defect in invasion. After $24 \mathrm{~h}$, LIT1 could be visualized by immunofluorescence around the periphery of wild-type, but not of $\Delta$ lit1, intracellular amastigotes (Fig. 4 E). Reinforcing

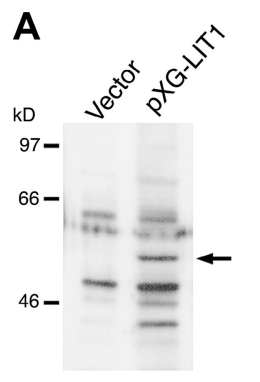

B

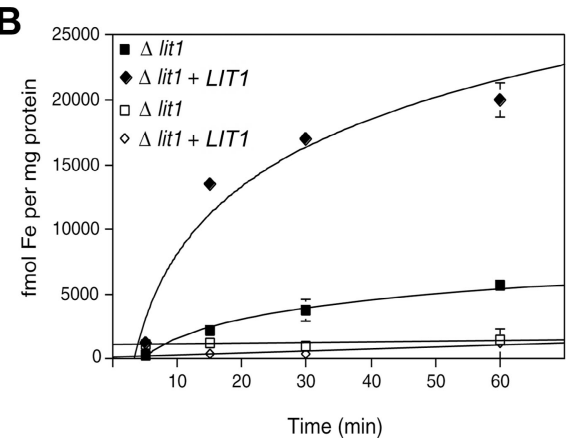

Figure 5. LIT1 expression promotes iron uptake. (A) $\Delta$ lit1 promastigotes were transfected with vector alone or with pXG-LIT1, lysed, and analyzed by SDS-PAGE, followed by Western blot with anti-LIT1 antibodies. The arrow points to the $\sim 50-\mathrm{kD}$ band detected only in parasites overexpressing LIT1. The bands detected on parasites transfected with vector alone correspond to unspecific background reactivity. (B) $\Delta$ lit1 promastigotes transfected with pXG-LIT1 were cultured at $32^{\circ} \mathrm{C}(\mathrm{pH}$ 5.5) to induce differentiation into axenic amastigotes, washed, and assayed for ${ }^{55} \mathrm{Fe}^{2+}$ uptake as described in Materials and methods. Closed squares and diamonds indicate incubation at $35^{\circ} \mathrm{C}$; open squares and diamonds indicate incubation at $4^{\circ} \mathrm{C}$ the conclusion that reactivity with the antibodies reflects an increase in LIT1 expression, a band of the predicted molecular mass $(\sim 50 \mathrm{kD})$ was detected in $\Delta$ lit 1 promastigotes transfected with the pXG-SAT episomal expression vector (28) carrying LIT1 (Fig. 5 A). When induced to differentiate into axenic amastigotes, ${ }^{55} \mathrm{Fe}^{2+}$ uptake activity was observed in these LIT1-overexpressing parasites (Fig. 5 B). These results directly demonstrate that LIT1 expression confers $\mathrm{Fe}^{2+}$ uptake activity to L. amazonensis amastigotes.

In addition to a normal capacity for entering BMmø and triggering initial expansion of the parasitophorous vacuole (Fig. 4 E), Dlit1 intracellular parasites also expressed P4, a specific marker of intracellular amastigotes (Fig. 6) (29). P4 was expressed by all $\Delta$ lit 1 intracellular amastigotes, regardless of whether the infections were initiated with axenic amastigotes (Fig. $6 \mathrm{~A}$ ) or with purified, infective metacyclic promastigotes (Fig. $6 \mathrm{~B}$ ). These results show that the LIT1 transporter is not required for the intracellular transition of recently internalized L. amazonensis into replicative amastigote forms.
A

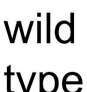

$\Delta$ lit1

B

wild
type
$\Delta$ lit1
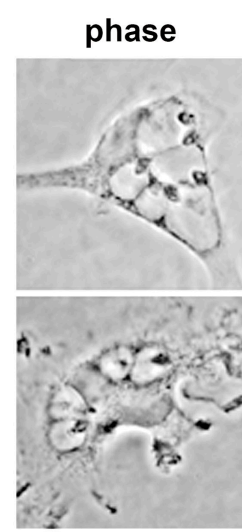

phase
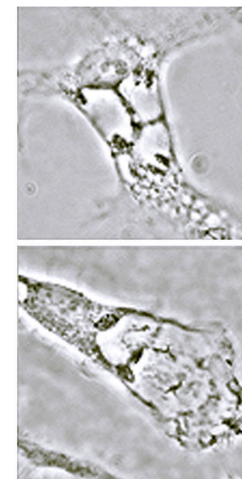

P4 antigen
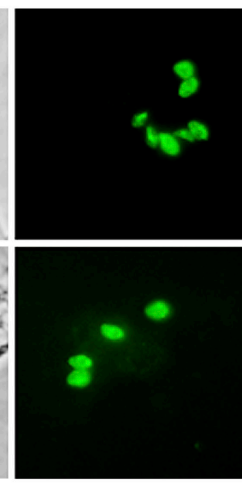

P4 antigen

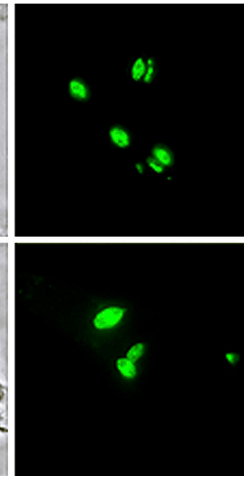

DAPI

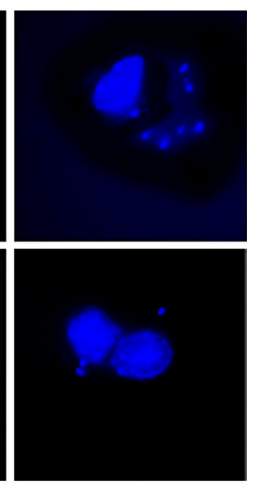

DAPI

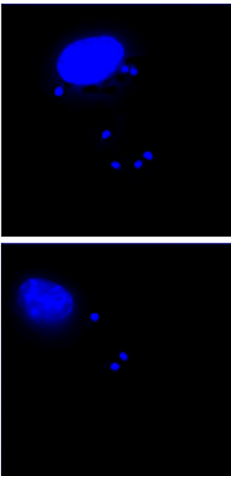

Figure 6. LIT1-null L. amazonensis differentiate normally into the intracellular mammalian stages. $B M m \emptyset$ were infected with wild-type or $\Delta$ lit1 parasites (A, axenic amastigotes; B, metacyclic promastigotes) for $1 \mathrm{~h}$, followed by washes, further incubation for $24 \mathrm{~h}$, fixation, permeabilization, and immunofluorescence with antibodies against the amastigotespecific antigen P4 (reference 29). (A and B, left) Phase contrast; ( $A$ and $B$, middle and right) fluorescence microscopy. Anti-P4 (green) and host and parasite DNA (blue) are shown. 
Next, we examined the capacity of L. amazonensis $\Delta$ lit 1 mutants for intracellular replication. In BMmø infected with wild-type axenic amastigotes, the number of intracellular parasites increased progressively between 24 and $72 \mathrm{~h}$ of incubation, as expected (Fig. 7 A, open columns). In contrast, there was no evidence for intracellular replication of $\Delta l i t 1$ amastigotes during the same period (Fig. 7 A, black-shaded columns). Importantly, the capacity for intracellular growth was completely restored in $\Delta$ lit 1 parasites expressing an episomally encoded wild-type copy of LIT1 ( $\Delta$ lit1 + LIT1) (Fig. 7 A, gray-shaded columns). Similar results were obtained when BMmø infections were performed using purified metacyclic promastigotes: wild-type parasites increased in number between 48 and $72 \mathrm{~h}$ after infection (Fig. $7 \mathrm{~B}$, open columns), whereas the numbers of intracellular $\Delta$ lit 1 parasites remained constant throughout this period (Fig. 7 B, black-shaded columns). Again, complementation with episomally encoded LIT1 completely restored the growth phenotype (Fig. 7 B, gray-shaded columns). In these experiments, the slower onset of $L$. amazonensis replication reflects the time period required for the intracellular differentiation of metacyclic promastigotes into replicative amastigotes. The results of both sets of experiments indicate the LIT1 transporter does not appear to be required for intracellular survival within the initial $72 \mathrm{~h}$, but it is essential for intracellular replication.

Next, we examined the replicative compartments containing wild-type, $\Delta l i t 1$, and $\Delta l i t 1+$ LIT1 parasites within macrophages. A remarkable feature of the intracellular lifestyle of Leishmania is that it thrives within acidified, hydrolase-rich compartments that share numerous properties with degradative lysosomes $(30,31)$. As expected, the lysosomal glycoprotein Lamp1 was present on the membrane of vacuoles surrounding wild-type L. amazonensis amastigotes at
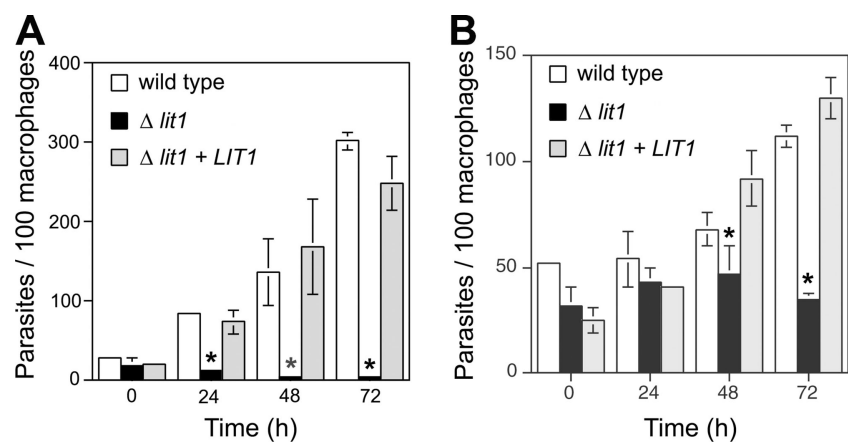

Figure 7. LIT1 is required for the intracellular replication of amastigotes. BMmø were infected for $1 \mathrm{~h}$ with wild-type, $\Delta$ lit1, and complemented $\Delta$ lit1 + LIT1 L. amazonensis axenic amastigotes (A) or metacyclic promastigotes (B), and the number of intracellular parasites was determined microscopically after further incubation for $0,24,48$ and $72 \mathrm{~h}$. The data represent the mean \pm SD of triplicate determinations and is representative of more than six independent experiments. The asterisks indicate significant differences between $\Delta$ lit1 and the wild type $(P<0.02)$. All values for infections with the complemented $\Delta$ lit1 + LIT1 line were not significantly different from the wild type $(P>0.05)$. early time points after invasion, as well as on the dramatically expanded compartments containing replicating parasites 48 and $72 \mathrm{~h}$ after infection (Fig. $8 \mathrm{~A}$ ). Lamp1 was also detected at all time points on the membrane of vacuoles surrounding $\Delta$ lit1 amastigotes, suggesting that LIT1 deletion does not affect biogenesis of the phagolysosomal compartment. However, marked differences were noted on the morphology of the vacuoles. By $48 \mathrm{~h}$ after infection, although some vacuole expansion was apparent, the overall size of the compartments containing $\Delta$ lit 1 parasites was much smaller and, generally, contained only one amastigote per vacuole. By $72 \mathrm{~h}$ after infection these compartments appeared shrunken, and some $\Delta$ lit1 parasites showed signs of degeneration (granulated appearance on phase-contrast and diffuse nuclear and kinetoplast staining; Fig. 8 B). In contrast, when $\Delta$ lit1 parasites were complemented with a wild-type copy of LIT1, by $48 \mathrm{~h}$ after infection the expansion of the Lamp1-positive vacuoles was completely restored, and each vacuole contained numerous amastigotes (Fig. 8 C). Interestingly, overexpression of LIT1 by transfection with the episomal plasmid seemed to interfere with the sustained expansion of the replicative compartments. By $72 \mathrm{~h}$ after infection, the large parasitophorous vacuoles containing $\Delta l i t 1+$ LIT1 parasites appeared somewhat collapsed, as indicated by the irregular staining pattern with antiLamp1 mAbs (Fig. 8 C). However, the complemented $\Delta$ lit1 + LIT1 intracellular amastigotes showed no signs of degeneration, which was consistent with the quantification assays that demonstrated a complete restoration in their capacity for intracellular replication (Fig. 7 A).

\section{LIT1 is required for the development of cutaneous lesions in mice}

In vivo infections with $L$. amazonensis lead to the development of cutaneous lesions, which are considered to be the result of parasite replication in tissue macrophages. To determine if the intracellular replication defect of LIT1-null amastigotes resulted in a loss of virulence in vivo, we injected high numbers of wild-type or $\Delta l i t 1$ metacyclic promastigotes into the footpads of BALB/c mice and followed lesion development for $>200 \mathrm{~d}$. Inoculation of up to $10^{6} \Delta$ lit 1 parasites yielded no pathology in $100 \%$ of the mice infected, with no footpad lesions detected for up to 6 mo in several independent experiments. In contrast, all mice inoculated with wildtype parasites developed progressive lesions within 2-3 wk and were killed by $42 \mathrm{~d}$ after infection. Confirming that LIT1 is required for lesion formation, complementation of the $\Delta$ lit1 mutant with either episomally encoded or a chromosome-integrated wild-type copy of the LIT1 gene restored lesion formation (Fig. 9 A). A several week delay was observed in the development of lesions in mice infected with the complemented strains when compared with rapid lesion development seen after inoculation of wild-type parasites. It is a common finding that Leishmania-complemented strains do not completely recover virulence, most likely because of the unregulated expression of the "add-back" proteins (32-34). In the experiment shown, one of five mice injected 
A

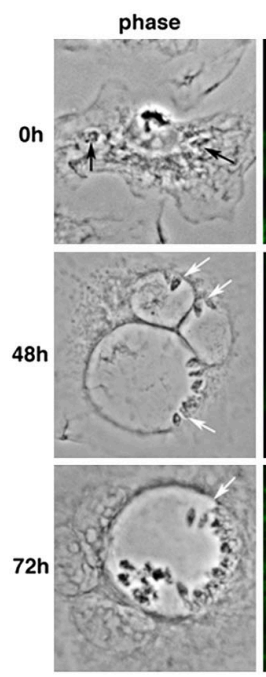

B

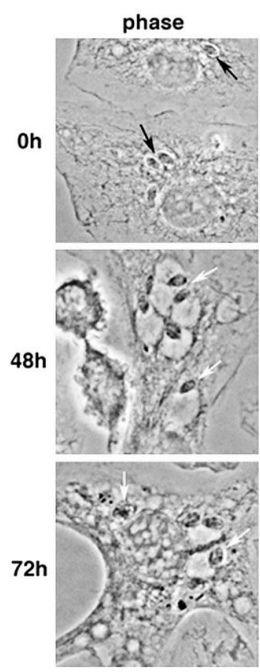

C

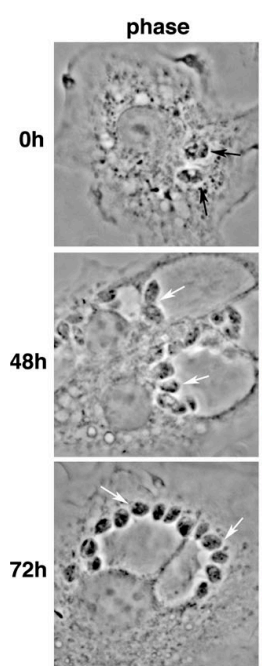

wild type

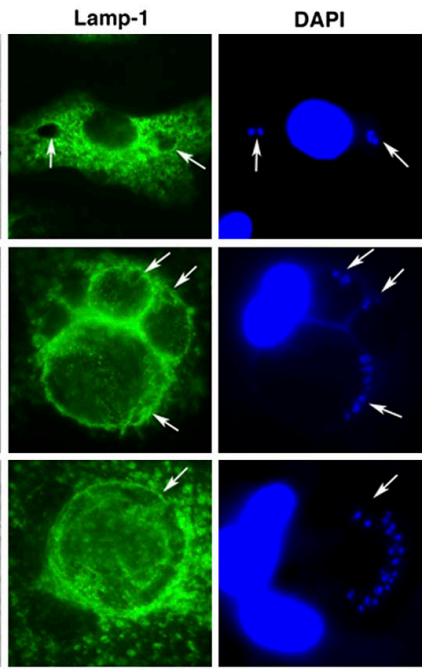

$\Delta$ lit1

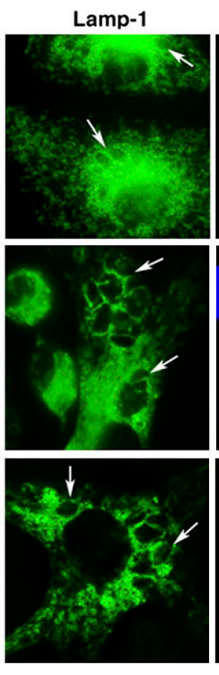

$\Delta$ lit1 + LIT1
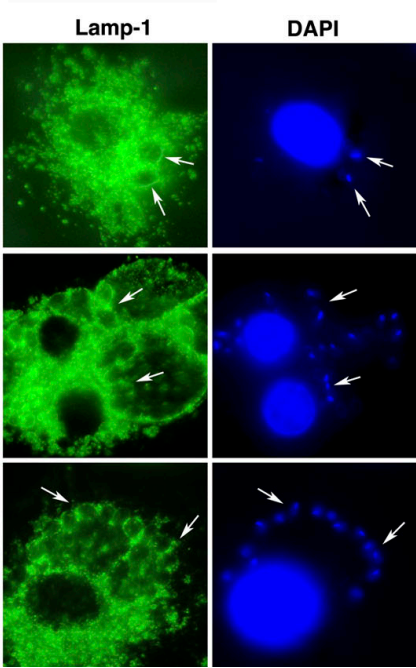

with $\Delta$ lit1 metacyclic promastigotes displayed measurable footpad swelling beginning at 13 wk that progressed slightly at $15 \mathrm{wk}$, when the mice were killed for quantitation of parasites in the footpad and in local draining lymph node (DLN). Interestingly, all of the mice inoculated with $\Delta$ lit 1 parasites harbored substantial numbers of viable amastigotes in the inoculation site (Fig. 9 B) and DLN (Fig. 9 C), including those without overt pathology. Nonetheless, there was at least a log-fold reduction in parasite loads in these tissues compared with mice infected with either of the complemented lines. These results are consistent with previous descriptions of in vivo persistence without pathology in Leishmania infections $(35,36)$. Further analysis of the $\Delta$ lit 1 promastigotes recovered from mice indicated that they retained the same properties of the original $\Delta$ lit1 inoculum, including hygromycin/neomycin resistance and no capacity for intracellular replication in macrophages (unpublished data).

\section{DISCUSSION}

Protozoan parasites belonging to the genus Leishmania are responsible for a spectrum of serious infections in humans, ranging from cutaneous lesions to a very severe visceralizing disease (37). Major gaps still exist in our understanding of the biology of different Leishmania species and how their different properties correlate with the various clinical forms of the disease. One common aspect of most Leishmania species, however, is their remarkable capacity for surviving and replicating within acidified, hydrolase-rich phagolysosomes of macrophages (30). In this study, we make an important step toward understanding the molecular mechanisms involved in adaptation to this harsh intracellular environment by identifying the first Leishmania membrane protein that functions intracellularly as a ferrous iron transporter. Our results show that the L. amazonensis LIT1 transporter is essential for intracellular replication in macrophages and for the development of pathogenic lesions in mice.

LIT1 was identified in the L. major genome database through its close homology to IRT1, a demonstrated high affinity $\mathrm{Fe}^{2+}$ transporter from $A$. thaliana. IRT1 was the first member to be identified in the ZIP family of metal transporters, which is now known to be present not only in plants but also in yeast, Drosophila, Caenorhabditis elegans, and humans (16). Null mutants of IRT1 in Arabidopsis have a severe

Figure 8. LIT1 regulates intracellular replication and parasitophorous vacuole expansion. BMmø were infected with wild-type (A), $\Delta$ lit 1 (B), or $\Delta$ lit1 + LIT1 (C) L. amazonensis axenic amastigotes for $1 \mathrm{~h}$ and either fixed immediately ( $0 \mathrm{~h}$ ) or further incubated for 48 or $72 \mathrm{~h}$. After permeabilization, immunofluorescence was performed with mAbs against Lamp1. (A-C, left) Phase contrast; ( $A-C$, middle and right) fluorescence microscopy. Antibodies to Lamp1 are labeled (green), and the host cell and parasite's DNA are stained (blue). Arrows point to intracellular parasites. The images show that although LIT1 overexpression ( $\Delta$ lit1 + LIT1 line) rescues the ability of $\Delta$ lit1 amastigotes to replicate intracellularly, expansion of Lamp1-positive membranes surrounding the parasitophorous vacuoles is not restored to wild-type levels. 

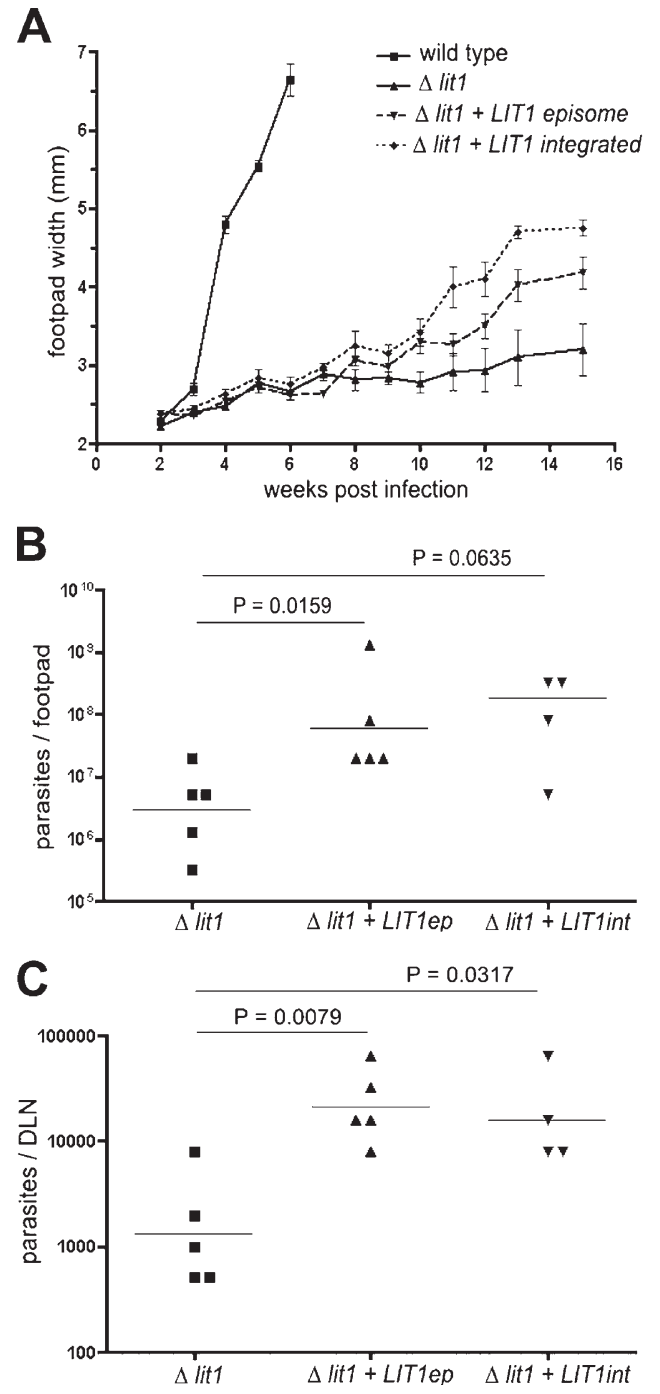

Figure 9. LIT1-null L. amazonensis are avirulent for mice. BALB/C mice were inoculated in the left hind footpad with $10^{6}$ ficoll-purified metacyclic promastigotes of L. amazonensis wild type, $\Delta$ lit1, $\Delta$ lit1 + LIT1 episomal (pXGSAT-LIT1), or $\Delta$ lit1 + LIT1 integrated (pIR1SAT-LIT1).

(A) Lesion development was quantitated by weakly caliper measurements. The data corresponds to the mean \pm SD of values obtained from five individual mice in each group. Parasite loads were determined in the footpad (B) or DLN (C) at 15 wk after challenge. Results shown represent parasite loads per footpad or DLN of individual mice, with geometric means and $p$-values between respective groups.

growth defect in normal soil, which is rescued by the exogenous application of iron (38). Additional reports demonstrated that iron is the preferred metal substrate for IRT1, although cadmium, cobalt, manganese, and zinc can also be transported $(17,22)$. As indicated by the preferential uptake of iron over cadmium when expressed in yeast (Fig. 3 B), LIT1 also functions as a divalent metal transporter with preference for iron.

The endocytic pathway in mammalian cells is thought to be iron poor, because acidification-induced release of $\mathrm{Fe}^{3+}$ from transferrin is followed by reduction to $\mathrm{Fe}^{2+}$ and translo- cation to the cytosol. It is well established that this function is performed by Nramp2 (also known as DCT1 or DMT1), the $\mathrm{pH}$-dependent divalent cation transporter localized on the membrane of endosomes $(9,10,39)$. Nramp1 (Slc11a1) is a close homologue of Nramp2, found on macrophage late endosomes/lysosomes. The similarity between Nramp1 and Nramp2 has led to the suggestion that both function as $\mathrm{pH}-$ dependent symporters, promoting the efflux of metal ions from endocytic compartments (4). However, different studies of this issue have reached contradictory conclusions, with some of the evidence indicating that Nramp1 may be an antiporter (40-43). Moreover, although Nramp1 was shown to be a $\mathrm{pH}$-dependent transporter capable of extruding $\mathrm{Mn}^{2+}$ from macrophage phagolysosomes (3), its postulated role in iron transport has not yet been directly demonstrated. Our findings provide new evidence in support of the notion that Nramp1 contributes to iron depletion from late endosomal compartments. Immunolocalization assays with antibodies specific for LIT1 failed to detect the protein in all extracellularly grown L. amazonensis life cycle stages and in parasites recently internalized by macrophages. However, a strong immunofluorescence signal was detected on intracellular amastigotes $12-24 \mathrm{~h}$ after infection of Nramp1-/- macrophages. Importantly, the intracellular expression of LIT1 appeared to be accelerated in macrophages derived from congenic Nramp $1^{+/+}$mice. Thus, similar to what was previously shown for Arabidopsis IRT1 (20) and other eukaryotic divalent cation transporters $(26,27)$, our results suggest that expression of the Leishmania LIT1 transporter is up-regulated by iron deprivation.

Although post-transcriptional regulation may also be involved, four lines of evidence suggest that LIT1 expression may be regulated posttranslationally by iron. First, LIT1 mRNA is present in axenic promastigotes and amastigotes, but no protein is detected with specific antibodies. Second, when overexpressed in promastigotes, GFP-LIT1 accumulates in intracellular compartments that may correspond to degradative lysosomes, in addition to the plasma membrane. Third, long-term residence in phagolysosomes, a compartment predicted to be iron poor, allows the detection of LIT1 with antibodies on the surface of amastigotes. Fourth, residence within phagolysosomes of Nramp1 ${ }^{+/+}$macrophages, postulated to be further depleted in iron, accelerates LIT1 expression. Collectively, these observations suggest that exposure to iron may induce LIT1 internalization and degradation, effectively removing the protein from the plasma membrane. This is a mechanism that was previously linked to the regulation of several eukaryotic divalent metal transporters $(20,26)$. Further characterization of this process in Leishmania will require the development of iron-free axenic culture conditions.

The exclusively intracellular expression pattern of LIT1 and the fact that it is dispensable for growth and differentiation in axenic culture suggests that alternative mechanisms for iron acquisition exist in the extracellular stages of L. amazonensis. This function may be fulfilled by the products of LmjF28.1330 
and/or LmjF33.3200, genes present in the L. major genome (chromosomes 28 and 33, respectively) that contain predicted ZIP metal permease domains and share reasonable similarity with putative metal transporters. The intracellular expression of LIT1 also highlights the fact that it has to compete with host late endosomal transporters for the same substrate, ferrous iron. The fact that episomal expression of LIT1 completely rescues the intracellular growth of LIT1-null parasites indicates that the transporter is active in the acidic $\mathrm{pH}$ of the phagolysosome. This scenario is in agreement with several earlier studies, which revealed acidic $\mathrm{pH}$ optima for the transport of glucose, amino acids, and polyamines by Leishmania amastigotes (44-46).

A unique property of $L$. amazonensis, when compared with other Leishmania species, is its capacity to induce the formation of dramatically expanded intracellular vacuoles, where the replicating amastigotes accumulate. It has been proposed that this enlargement of the parasitophorous vacuole may be linked to the unique ability of $L$. amazonensis to survive in IFN- $\gamma$-activated macrophages (47). Little is known about how these enlarged compartments are maintained and how expansion affects the concentration of microbicidal products and nutrients within the vacuoles. Interestingly, although complementation of LIT1-null L. amazonensis with wild-type LIT1 restored their ability to replicate within macrophages (Fig. 7 A, $\Delta$ lit $1+$ LIT1 column), the swollen parasitophorous vacuoles appeared partially collapsed at later times after infection (Fig. $8 \mathrm{C}, 72 \mathrm{~h}$ ). This may be related to the overexpression of LIT1 when introduced in an episomal vector, suggesting that vacuole expansion and maintenance may be affected by the rate by which $\mathrm{Fe}^{2+}$ is translocated into the parasite versus $\mathrm{Fe}^{2+}$ efflux from the vacuole.

Our results clearly show that the block in intracellular replication of L. amazonensis lacking the LIT1 transporter is not caused by defects in invasion, early survival within macrophages, or in differentiation into replicative amastigotes. These findings reinforce previous suggestions that the ability to replicate as amastigotes is the most important requirement for virulence in Leishmania (48). Indeed, mutations in genes involved in macrophage infection and early survival (but not in growth as amastigotes) lead only to attenuation in virulence and delayed lesion formation $(34,49)$. On the other hand, mutants incapable of growing in macrophages, such as the L. major $\operatorname{lpg} 2^{-}$lacking phosphoglycans (36) and the L. amazonensis $\Delta$ lit1 described in this paper, are avirulent. Remarkably, both $\operatorname{lpg} 2^{-}$L. major and $\Delta$ lit1 L. amazonensis can still persist in vivo without causing pathology (36). It is important to note, however, that the $\lg p 2^{-}$persisting population can give rise to compensatory mutants with restored virulence despite their consistent lack of phosphoglycan expression (48). No evidence for such reversal of the avirulent phenotype was seen so far in our studies, suggesting that secondary mutations may not be able to compensate for the absence of LIT1. In future studies, it will be of great interest to take advantage of the defined iron transport defect of $\Delta$ lit1 L. amazonensis to pursue the cellular basis of the compartment where avirulent parasites persist in host tissues.

\section{MATERIALS AND METHODS}

Parasites. D.L. Sacks provided the L. amazonensis IFLA/BR/67/PH8 strain. Promastigotes were maintained in vitro at $26^{\circ} \mathrm{C}$ in $\mathrm{M} 199$ (pH 7.4; Invitrogen) supplemented with $20 \%$ heat-inactivated FBS, $5 \%$ penicillin-streptomycin, $0.1 \%$ hemin $(25 \mathrm{mg} / \mathrm{ml}$ in $50 \%$ triethanolamine), $10 \mathrm{mM}$ adenine ( $\mathrm{pH} 7.5)$, and $5 \mathrm{mM}$ L-glutamine (M199/S). Axenic amastigotes were cultured at $32^{\circ} \mathrm{C}$ in the same medium supplemented with $0.25 \%$ glucose, $0.5 \%$ trypticase, and $40 \mathrm{mM} \mathrm{Na}$ succinate ( $\mathrm{pH} 4.5$ ). Metacyclic promastigotes were purified from 7 -d-old promastigote stationary phase cultures using the mAb 3A1, which specifically agglutinates $L$. amazonensis procyclic promastigotes but not metacyclics $(25,50)$. Parasites were washed twice with PBS, resuspended at $2.5 \times 10^{8}$ parasites $/ \mathrm{ml}$ in $0.5 \mathrm{ml}$ PBS containing a 1:500 dilution of $3 \mathrm{~A} 1 \mathrm{ascites}$ for $30 \mathrm{~min}$, and centrifuged at $250 \mathrm{~g}$ for $5 \mathrm{~min}$. Nonagglutinated parasites in the supernatant were washed twice, counted, and used for infection of macrophages.

Identification of LIT1 in the L. amazonensis genome. BLAST homology searches of the L. major database identified two identical genes in tandem, LmjF31.3060 and LmjF31.3070, that shared 30\% identity and 53\% similarity to the $A$. thaliana iron transporter IRT1. The following primers were used to amplify the corresponding 1.3-kb gene from genomic DNA of L. amazonensis: forward, 5'-GGATCCATGGAGACGGCGAAACTG-3'; and reverse, 5'-GGATCCCTACAGCCAGTTGCCCAC-3' (underlined sequences indicate added BamHI sites). The PCR product was cloned into the pCR2.1TOPO vector (Invitrogen) to generate a pCR-LIT1 plasmid, and the correct coding sequence was confirmed by sequencing.

Gene deletion constructs. The gene deletion constructs (LaKOLITHyg and LaKOLITNeo) required for sequential deletion of both alleles of the LIT1 genes were based on the Leishmania expression vectors pXG-hyg and pXG-neo (courtesy of S. Beverley, Washington University, St. Louis, MO) (28). A 2-kb flanking sequence upstream of the LaLIT1-1 open reading frame (ORF) was PCR amplified using the following primers: forward, 5 ' GGAGYGCTTGTACGACCTCC-3' ${ }^{\prime}$; and reverse, $5^{\prime}$ '-GGATCCCGGGAGCAAGAGGGAGATAGAG-3'. A 1.2-kb flanking sequence downstream of the LaLIT1-2 ORF was amplified using the following primers: forward, 5'-GGATCCCGGGAGAGCGCATTGACTTGGT-3'; and reverse, 5'GCTCTGCATATCTGCCATAC-3'. BamHI and SmaI restriction sites were created in both fragments and used for cloning into the pCR2.1 vector (Invitrogen). The upstream fragment was excised from the vector and cloned into the BamHI-linearized construct containing the downstream sequence. To generate the deletion constructs, the spanning regions containing DHFRHyg (or Neo)-TS were PCR amplified from the pXG-based vectors and ligated to the described construct, linearized by SmaI digestion. Plasmid DNA from each gene-targeting construct was digested with BamHI and XhoI to release the integrating fragments, and the linearized gene deletion constructs were gel purified.

Transfection and generation of LIT1-null L. amazonensis. Mid-log L. amazonensis promastigotes were collected by centrifugation, washed once with PBS and once with ice-cold electroporation buffer $(21 \mathrm{mM}$ Hepes, $\mathrm{pH}$ 7.5, $0.7 \mathrm{mM} \mathrm{NaH}{ }_{2} \mathrm{PO}_{4}, 137 \mathrm{mM} \mathrm{NaCl}, 5 \mathrm{mM} \mathrm{KCl}$, and $6 \mathrm{mM}$ glucose), and resuspended at $10^{8}$ cells $/ \mathrm{ml}$. A volume of $0.4 \mathrm{ml}$ was mixed with $10 \mu \mathrm{g}$ DNA and placed into a $0.2 \mathrm{ml}$ gap cuvette (Bio-Rad Laboratories). The cuvette was chilled on ice for $10 \mathrm{~min}$, electroporated using a Cellpulser (Bio-Rad Laboratories) set at $450 \mathrm{~V}, 500 \mu \mathrm{F}$, and returned to ice for $10 \mathrm{~min}$, followed by transfer of the promastigotes to $10 \mathrm{ml}$ of growth medium and incubation at $26^{\circ} \mathrm{C}$. After $2 \mathrm{~d}$, promastigotes were plated on agar dishes $(2 \%$ agar in complete promastigote growth medium) containing the appropriate drug for selection and incubated at $27^{\circ} \mathrm{C}$. Colonies visible after $15 \mathrm{~d}$ were picked and tested for integration. For generation of the $\Delta$ lit 1 knockout, the region containing the two LIT1 genes was replaced sequentially on both alleles by the hygromycin B phosphotransferase $(\mathrm{H} y g)$ and neomycin phosphotransferase $(\mathrm{Neo})$ genes, which confer resistance to the antibiotics hygromycin B and G418, respectively. $100 \mu \mathrm{g} / \mathrm{ml}$ hygromycin B and/or $50 \mu \mathrm{g} / \mathrm{ml}$ 
G418 were added to the medium used to expand the isolated colonies after each round of homologous gene targeting. Southern blots were performed to determine integration of the selectable markers at the LIT1 locus. The homozygous $\Delta$ lit 1 strain was cultured in complete promastigote growth medium containing $100 \mu \mathrm{g} / \mathrm{ml}$ hygromycin B and $50 \mu \mathrm{g} / \mathrm{ml} \mathrm{G418.} \mathrm{Comple-}$ mentation of $\Delta$ lit 1 parasites with wild-type LIT1 was achieved by transfection of promastigotes with the episomal vector pXGSAT-LIT1 (generated by cloning a BamHI fragment containing the LIT1 ORF into the pXGSAT vector [28]) or linearized pIR1SAT-LIT1 (generated by inserting the LIT1 ORF into the SmaI site of the pIR1SAT vector that promotes integration into the ribosomal SSU locus [provided by S. Beverley]). Agar-grown clones resistant to $50 \mu \mathrm{g} / \mathrm{ml}$ nourseothricin were selected for further characterization.

For Northern blots, $10 \mu \mathrm{g}$ of total RNA extracted from L. amazonensis amastigotes and promastigotes (RNeasy Mini Kit; QIAGEN) were loaded onto $1.2 \% \mathrm{MOPS} /$ formaldehyde agarose gels, transferred to a nylon membrane, and prehybridized for $2 \mathrm{~h}$ at $42^{\circ} \mathrm{C}$ in $10 \mathrm{ml}$ of $5 \times$ Denhardt's solution $/ 5 \times$ standard sodium phosphate with EDTA/1\% SDS with $200 \mu \mathrm{g} / \mathrm{ml}$ salmon sperm DNA. Hybridization was performed overnight at $42^{\circ} \mathrm{C}$ in $10 \mathrm{ml}$ of the same buffer containing 50\% formamide and the ${ }^{32} \mathrm{P}$-labeled LIT1 probe. Filters were washed and exposed at $-70^{\circ} \mathrm{C}$ for autoradiography.

${ }^{55} \mathrm{Fe}$ incorporation assays. Axenic amastigotes were washed twice with PBS, resuspended in uptake buffer $(140 \mathrm{mM} \mathrm{NaCl}, 5 \mathrm{mM} \mathrm{KCl}, 1 \mathrm{mM}$ $\mathrm{CaCl}_{2}, 0.09 \%$ glucose, $\left.10 \mathrm{mM} \mathrm{MES}, \mathrm{pH} 5.5\right)$ at $5 \times 10^{8}$ parasites $/ \mathrm{ml}$, and placed in 100- $\mu$ l aliquots in microcentrifuge tubes. Ascorbic acid (to reduce $\mathrm{Fe}^{3+}$ to $\mathrm{Fe}^{2+}$ ) was added to the uptake buffer to a final concentration of $50 \mu \mathrm{M}$ from a $1-\mathrm{mM}$ freshly made solution. To maintain ${ }^{55} \mathrm{Fe}(82.69 \mathrm{mCi} / \mathrm{mg}$, $39 \mathrm{mCi} / \mathrm{ml}$; PerkinElmer,) in solution for uptake, a $\mathrm{FeCl}_{3}$-nitrilotriacetic acid (Fe-NTA) solution was prepared at a 1:50 molar ratio. To initiate iron uptake, $100 \mu{ }^{55} \mathrm{Fe}-\mathrm{NTA}\left(2 \mu \mathrm{M}{ }^{55} \mathrm{Fe}\right)$ was added to the cell suspension, and the samples were incubated at $35^{\circ} \mathrm{C}$ or $4^{\circ} \mathrm{C}$ for various time intervals. At the end of the incubation period, parasites were washed three times with PBS, the pellets were resuspended in $100 \mu \mathrm{lBS}$, and the cell-associated radioactivity was counted in a liquid scintillation counter (Wallac 1409; PerkinElmer). The protein concentration of the samples was determined by a bicinchoninic acid assay (Pierce Chemical Co.).

LIT1 expression in yeast. The LIT1 ORF was placed under the control of the ADH promoter for overexpression in $S$. cerevisiae by cloning into the pAD4M (LEU2 ${ }^{+}$) or p426 $\left(\mathrm{URA}^{+}\right.$) vectors $(51)$ at the SmaI or BamHI sites, respectively. The $\Delta$ fet 3 fet 4 yeast strain (provided by M.L. Guerinot, Dartmouth College, Hanover, NH) $(21,22)$ was transformed with p426LIT1 and selected on minimal essential plates without uracil before streaking on rich media (YPD) with or without the iron chelator BPS (final concentrations of 20 or $40 \mu \mathrm{M}$ ). A wild-type strain (W303) was transformed with pAD4M-LIT1 and selected on minimal essential plates without leucine. The transformed yeast colonies were tested for the ability to grow at $30^{\circ} \mathrm{C}$ for $5 \mathrm{~d}$ on plates containing $100 \mu \mathrm{M} \mathrm{CdCl}_{3}$, or $100 \mu \mathrm{M}$ $\mathrm{CdCl}_{3}$ and $100 \mu \mathrm{M} \mathrm{FeCl}_{3}$.

Infection of primary bone marrow macrophages. BMmø isolated from C57/BL6 mice (Charles River Laboratories) were prepared as described previously (52), seeded onto 24-well plates containing coverslips at a density of $7 \times 10^{4}$ cells per well, and incubated overnight in RPMI 1640 with $10 \%$ FBS containing $5 \% \mathrm{~L}$ cell supernatant (as a source of $\mathrm{M}-\mathrm{CSF}$ ) at $37^{\circ} \mathrm{C}$ and $5 \% \mathrm{CO}_{2}$. Attached BMmø were washed with fresh RPMI 1640 and infected with $1.4 \times 10^{5}$ axenic amastigotes or $7 \times 10^{5}$ metacyclic promastigotes per well in $0.5 \mathrm{ml}$ RPMI 1640 with 2\% FBS (multiplicity of infection $=2$ and 10 for amastigotes and metacyclic promastigotes, respectively). BMmø isolated from C57BL/ 10ScSn $\left(\mathrm{Nramp}^{+/+}\right)$or B10.L-Lsh $\left(\mathrm{Nramp}^{-/-}\right)$congenic mice (provided by J. Blackwell, Cambridge University, Cambridge, UK) (53) were cultured in DMEM with $10 \%$ FBS containing $15 \% \mathrm{~L}$ cell supernatant for $6 \mathrm{~d}$, followed by replacement of half the medium with DMEM with $10 \%$ FBS at day 7 and infection on day 8 in DMEM with $10 \%$ FBS (54). After $1 \mathrm{~h}$ at $34^{\circ} \mathrm{C}$ and $5 \%$ $\mathrm{CO}_{2}$, free parasites were removed by three washes with PBS, and the cultures were further incubated in for various periods of time, followed by fixation with
$2 \%$ paraformaldehyde (PFA) and staining with DAPI. The number of intracellular parasites (identified through the characteristic kinetoplast DNA stained with DAPI and localization within enlarged vacuoles by phase contrast) was determined at 100× with a microscope (Axiovert 200; Carl Zeiss MicroImaging, Inc.) in a minimum of 400 macrophages per coverslip in triplicate, and the data were expressed as the total number of intracellular parasites per 100 macrophages. The data were analyzed for statistical significance using an unpaired Student's $t$ test $(\mathrm{P}<0.05$ was considered significant).

Fluorescence microscopy. To construct a GFP-LIT1 gene fusion, the LIT1 ORF was cloned into the $\mathrm{pXG}-\mathrm{GFP}_{2}{ }^{+}$vector (courtesy of S. Beverley) (28), which drives the expression in Leishmania of proteins fused to GFP at the amino terminus. Transfected $L$. amazonensis promastigote clones ex-

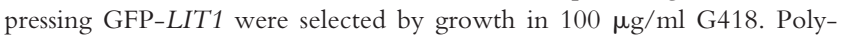
clonal antibodies against LIT1 were generated by immunizing a rabbit with the first 15 amino acids of LIT1 (METAKLSVEASTRHL) coupled to keyhole limpet hemocyanin (MBS cross-linking reagent; Pierce Chemical Co.), and affinity purified using the cognate peptide coupled to Affigel 15 (Bio-Rad Laboratories). For immunofluorescence, BMmø infected with the various $L$. amazonensis strains (wild-type, $\Delta l i t 1$, and $\Delta l i t 1+$ LIT1) were fixed with $2 \%$ PFA, blocked with $50 \mathrm{mM} \mathrm{NH}_{4} \mathrm{Cl}$ and $2 \%$ goat serum in PBS, permeabilized in $0.1 \mathrm{mg} \mathrm{ml}^{-1}$ saponin, and incubated for $1 \mathrm{~h}$ at room temperature with rabbit polyclonal antibodies against LIT1, P4 (courtesy of D. McMahon-Pratt, Yale University, New Haven, CT) (29), or mAbs against mouse lysosomal-associated membrane protein 1 Lamp1 (1D4B; Developmental Studies Hybridoma Bank). After incubation with Alexaconjugated goat anti-rabbit or -mouse IgG (Invitrogen), coverslips were mounted in antifade reagent (ProLong; Invitrogen) and examined by a fluorescence microscope (Axiovert 200) equipped with a CCD camera (CoolSNAP HQ; Photometrics) controlled by software (MetaMorph; Molecular Devices Corporation).

In vivo virulence and persistence assays. Female $\mathrm{BALB} / \mathrm{c}$ mice were injected in the left hind footpad with $10^{6}$ ficoll gradient-purified metacyclic promastigotes of L. amazonensis (55), and lesion progression was followed by blinded weekly measurements with a caliper. The total number of parasites in the injected footpad and local DLN $15 \mathrm{wk}$ after infection was estimated by a limiting dilution assay, as previously described (56). Infected footpad tissue was prepared after removal of the toes and bones by incubation for $2 \mathrm{~h}$ at $37^{\circ} \mathrm{C}$ in DME containing $100 \mathrm{U} / \mathrm{ml}$ penicillin, $100 \mu \mathrm{g} / \mathrm{ml}$ streptomycin, and $50 \mu \mathrm{g} / \mathrm{ml}$ Liberase CI enzyme blend (Boehringer). Footpad tissues were ground in a Medimachine (Beckton Dickinson). Popliteal lymph nodes were removed and mechanically dissociated using a pellet pestle in $100 \mu \mathrm{l}$ DME containing $100 \mathrm{U} / \mathrm{ml}$ penicillin and $100 \mu \mathrm{g} / \mathrm{ml}$ streptomycin medium. Tissue homogenates of both infected tissues and DLNs were filtered using a $70-\mu \mathrm{m}$ pore size cell strainer (Falcon Products, Inc.). Recovered cells were serially diluted in a 96-well flatbottom microtiter plate containing biphasic medium prepared using $50 \mu 1$ NNN medium containing $20 \%$ defibrinated rabbit blood and overlaid with $100 \mu \mathrm{l}$ M199/S medium. The number of viable parasites was determined from the highest dilution at which promastigotes could be grown out after $7-10 \mathrm{~d}$ of incubation at $26^{\circ} \mathrm{C}$. Statistical significance between means of various groups was determined using a twotailed $t$ test for independent samples.

Online supplemental material. The expression of LIT1 by L. amazonensis amastigotes in BMmø from C57BL/10ScSn (Nramp1 ${ }^{-/-}$) or B10.L-Lsh $\left(\mathrm{Nramp}^{+/+}\right)$mice was examined by immunofluorescence with specific antibodies. LIT1 was detected earlier (6 h after infection) in B10.L-Lsh BMmø, suggesting that iron depletion from the parasitophorous vacuole mediated by the Nramp1 transporter accelerates LIT1 expression.

Fig. S1 shows Nramp1-dependent expression of LIT1 by intracellular L. amazonensis. Randomly acquired, independent microscopic fields showing immunofluorescence of $\mathrm{C} 57 \mathrm{BL} / 10 \mathrm{ScSn}\left(\mathrm{Nramp1}^{-/-}\right)$or B10.L-Lsh $\left(\mathrm{Nramp}^{+/+}\right.$) BMmø after 1 (A) or 6 (B) h of infection with L. amazonensis axenic amastigotes. LIT1 is detected after $6 \mathrm{~h}$ of infection in $\mathrm{Nramp} 1^{+/+}$ 
BMmø, whereas expression levels remain low in Nramp1 $1^{-/-}$BMmø at 1 and $6 \mathrm{~h}$ after infection. Antibodies to LIT1 are labeled in green, and the host cell and parasite's DNA are stained in blue (DAPI). Arrows point to infected macrophages. The images were acquired and enhanced for contrast under identical conditions. Online supplemental material is available at http://www.jem.org/cgi/content/full/jem.20060559/DC1.

We thank Dr. Jenefer Blackwell for her gift of C57BL/10ScSn and B10.L-Lsh congenic mice, as well as for helpful discussions. We are also grateful to Drs. Steve Beverley, Diane McMahon-Pratt, and Mary Lou Guerinot for their gifts of plasmids, antibodies, or strains, as well as Jude Wilson for a critical reading of the manuscript.

This work was supported by National Institutes of Health grant R37Al34867 to N.W. Andrews.

The authors have no conflicting financial interests.

\section{Submitted: 13 March 2006}

Accepted: 31 August 2006

\section{REFERENCES}

1. Weinberg, E.D. 1999. The role of iron in protozoan and fungal infectious diseases. J. Eukaryot. Microbiol. 46:231-238.

2. Blackwell, J.M., T. Goswami, C.A. Evans, D. Sibthorpe, N. Papo, J.K. White, S. Searle, E.N. Miller, C.S. Peacock, H. Mohammed, and M. Ibrahim. 2001. SLC11A1 (formerly NRAMP1) and disease resistance. Cell. Microbiol. 3:773-784.

3. Jabado, N., A. Jankowski, S. Dougaparsad, V. Picard, S. Grinstein, and P. Gros. 2000. Natural resistance to intracellular infections: natura resistance-associated macrophage protein 1 (Nramp1) functions as a $\mathrm{pH}-$ dependent manganese transporter at the phagosomal membrane. J. Exp. Med. 192:1237-1248.

4. Forbes, J.R., and P. Gros. 2001. Divalent-metal transport by NRAMP proteins at the interface of host-pathogen interactions. Trends Microbiol. 9:397-403.

5. Paramchuk, W.J., S.O. Ismail, A. Bhatia, and L. Gedamu. 1997. Cloning, characterization and overexpression of two iron superoxide dismutase cDNAs from Leishmania chagasi: role in pathogenesis. Mol. Biochem. Parasitol. 90:203-221.

6. Wilson, M.E., and B.E. Britigan. 1998. Iron uptake by parasitic protozoa. Parasitol. Today. 14:348-353.

7. Schaible, U.E., and S.H. Kaufmann. 2004. Iron and microbial infection. Nat. Rev. Microbiol. 2:946-953.

8. Kaplan, J. 2002. Mechanisms of cellular iron acquisition: another iron in the fire. Cell. 111:603-606.

9. Ohgami, R.S., D.R. Campagna, E.L. Greer, B. Antiochos, A. McDonald, J. Chen, J.J. Sharp, Y. Fujiwara, J.E. Barker, and M.D. Fleming. 2005. Identification of a ferrireductase required for efficient transferrin-dependent iron uptake in erythroid cells. Nat. Genet. 37:1264-1269.

10. Fleming, M.D., M.A. Romano, M.A. Su, L.M. Garrick, M.D. Garrick, and N.C. Andrews. 1998. Nramp2 is mutated in the anemic Belgrade (b) rat: evidence of a role for Nramp2 in endosomal iron transport. Proc. Natl. Acad. Sci. USA. 95:1148-1153.

11. Wooldridge, K.G., and P.H. Williams. 1993. Iron uptake mechanisms of pathogenic bacteria. FEMS Microbiol. Rev. 12:325-348.

12. Wilson, M.E., R.W. Vorhies, K.A. Andersen, and B.E. Britigan. 1994. Acquisition of iron from transferrin and lactoferrin by the protozoan Leishmania chagasi. Infect. Immun. 62:3262-3269.

13. Steverding, D., Y.D. Stierhof, H. Fuchs, R. Tauber, and P. Overath. 1995. Transferrin-binding protein complex is the receptor for transferrin uptake in Trypanosoma brucei. J. Cell Biol. 131:1173-1182.

14. Borges, V.M., M.A. Vannier-Santos, and W. de Souza. 1998. Subverted transferrin trafficking in Leishmania-infected macrophages. Parasitol. Res. 84:811-822.

15. Wilson, M.E., T.S. Lewis, M.A. Miller, M.L. McCormick, and B.E. Britigan. 2002. Leishmania chagasi: uptake of iron bound to lactoferrin or transferrin requires an iron reductase. Exp. Parasitol. 100:196-207.

16. Guerinot, M.L. 2000. The ZIP family of metal transporters. Biochim. Biophys. Acta. 1465:190-198.
17. Rogers, E.E., D.J. Eide, and M.L. Guerinot. 2000. Altered selectivity in an Arabidopsis metal transporter. Proc. Natl. Acad. Sci. USA. 97:12356-12360.

18. Mullin, K.A., B.J. Foth, S.C. Ilgoutz, J.M. Callaghan, J.L. Zawadzki, G.I. McFadden, and M.J. McConville. 2001. Regulated degradation of an endoplasmic reticulum membrane protein in a tubular lysosome in Leishmania mexicana. Mol. Biol. Cell. 12:2364-2377.

19. Ghedin, E., A. Debrabant, J.C. Engel, and D.M. Dwyer. 2001. Secretory and endocytic pathways converge in a dynamic endosomal system in a primitive protozoan. Traffic. 2:175-188.

20. Connolly, E.L., J.P. Fett, and M.L. Guerinot. 2002. Expression of the IRT1 metal transporter is controlled by metals at the levels of transcript and protein accumulation. Plant Cell. 14:1347-1357.

21. Dix, D.R., J.T. Bridgham, M.A. Broderius, C.A. Byersdorfer, and D.J. Eide. 1994. The FET4 gene encodes the low affinity Fe(II) transport protein of Saccharomyces cerevisiae. J. Biol. Chem. 269:26092-26099.

22. Eide, D., M. Broderius, J. Fett, and M.L. Guerinot. 1996. A novel ironregulated metal transporter from plants identified by functional expression in yeast. Proc. Natl. Acad. Sci. USA. 93:5624-5628.

23. Gietz, R.D., and R.H. Schiestl. 1991. Applications of high efficiency lithium acetate transformation of intact yeast cells using single-stranded nucleic acids as carrier. Yeast. 7:253-263.

24. Jensen, L.T., and V.C. Culotta. 2002. Regulation of Saccharomyces cerevisiae FET4 by oxygen and iron. J. Mol. Biol. 318:251-260.

25. Pinto-da-Silva, L.H., P. Fampa, D.C. Soares, S.M. Oliveira, T. SoutoPadron, and E.M. Saraiva. 2005. The 3A1-La monoclonal antibody reveals key features of Leishmania $(L)$ amazonensis metacyclic promastigotes and inhibits procyclics attachment to the sand fly midgut. Int. J. Parasitol. 35:757-764.

26. Felice, M.R., I. De Domenico, L. Li, D.M. Ward, B. Bartok, G. Musci, and J. Kaplan. 2005. Post-transcriptional regulation of the yeast high affinity iron transport system. J. Biol. Chem. 280:22181-22190.

27. Canonne-Hergaux, F., S. Gruenheid, P. Ponka, and P. Gros. 1999. Cellular and subcellular localization of the Nramp2 iron transporter in the intestinal brush border and regulation by dietary iron. Blood. 93:4406-4417.

28. Ha, D.S., J.K. Schwarz, S.J. Turco, and S.M. Beverley. 1996. Use of the green fluorescent protein as a marker in transfected Leishmania. Mol. Biochem. Parasitol. 77:57-64.

29. Kar, S., L. Soong, M. Colmenares, K. Goldsmith-Pestana, and D. McMahon-Pratt. 2000. The immunologically protective P-4 antigen of Leishmania amastigotes. A developmentally regulated single strandspecific nuclease associated with the endoplasmic reticulum. J. Biol. Chem. 275:37789-37797.

30. Antoine, J.C., E. Prina, T. Lang, and N. Courret. 1998. The biogenesis and properties of the parasitophorous vacuoles that harbour Leishmania in murine macrophages. Trends Microbiol. 6:392-401.

31. Courret, N., C. Frehel, N. Gouhier, M. Pouchelet, E. Prina, P. Roux, and J.C. Antoine. 2002. Biogenesis of Leishmania-harbouring parasitophorous vacuoles following phagocytosis of the metacyclic promastigote or amastigote stages of the parasites. J. Cell Sci. 115:2303-2316.

32. Mottram, J.C., A.E. Souza, J.E. Hutchison, R. Carter, M.J. Frame, and G.H. Coombs. 1996. Evidence from disruption of the lmcpb gene array of Leishmania mexicana that cysteine proteinases are virulence factors. Proc. Natl. Acad. Sci. USA. 93:6008-6013.

33. Wiese, M. 1998. A mitogen-activated protein (MAP) kinase homologue of Leishmania mexicana is essential for parasite survival in the infected host. EMBO J. 17:2619-2628.

34. Spath, G.F., L. Epstein, B. Leader, S.M. Singer, H.A. Avila, S.J. Turco, and S.M. Beverley. 2000. Lipophosphoglycan is a virulence factor distinct from related glycoconjugates in the protozoan parasite Leishmania major. Proc. Natl. Acad. Sci. USA. 97:9258-9263.

35. Sacks, D., and N. Noben-Trauth. 2002. The immunology of susceptibility and resistance to Leishmania major in mice. Nat. Rev. Immunol. 2:845-858.

36. Spath, G.F., L.F. Lye, H. Segawa, D.L. Sacks, S.J. Turco, and S.M. Beverley. 2003. Persistence without pathology in phosphoglycandeficient Leishmania major. Science. 301:1241-1243. 
37. McMahon-Pratt, D., and J. Alexander. 2004. Does the Leishmania major paradigm of pathogenesis and protection hold for New World cutaneous leishmaniases or the visceral disease? Immunol. Rev. 201:206-224.

38. Vert, G., N. Grotz, F. Dedaldechamp, F. Gaymard, M.L. Guerinot, J.F. Briat, and C. Curie. 2002. IRT1, an Arabidopsis transporter essential for iron uptake from the soil and for plant growth. Plant Cell. 14:1223-1233.

39. Picard, V., G. Govoni, N. Jabado, and P. Gros. 2000. Nramp 2 (DCT1/ DMT1) expressed at the plasma membrane transports iron and other divalent cations into a calcein-accessible cytoplasmic pool. J. Biol. Chem. 275:35738-35745.

40. Gomes, M.S., and R. Appelberg. 1998. Evidence for a link between iron metabolism and Nramp1 gene function in innate resistance against Mycobacterium avium. Immunology. 95:165-168.

41. Zwilling, B.S., D.E. Kuhn, L. Wikoff, D. Brown, and W. Lafuse. 1999 Role of iron in Nramp1-mediated inhibition of mycobacterial growth Infect. Immun. 67:1386-1392.

42. Kuhn, D.E., B.D. Baker, W.P. Lafuse, and B.S. Zwilling. 1999. Differential iron transport into phagosomes isolated from the RAW264.7 macrophage cell lines transfected with Nramp1Gly169 or Nramp1Asp169. J. Leukoc. Biol. 66:113-119.

43. Goswami, T., A. Bhattacharjee, P. Babal, S. Searle, E. Moore, M. Li, and J.M. Blackwell. 2001. Natural-resistance-associated macrophage protein 1 is an $\mathrm{H}+$ /bivalent cation antiporter. Biochem. J. 354:511-519.

44. Burchmore, R.J., and D.T. Hart. 1995. Glucose transport in amastigotes and promastigotes of Leishmania mexicana mexicana. Mol. Biochem. Parasitol. 74:77-86.

45. Mazareb, S., Z.Y. Fu, and D. Zilberstein. 1999. Developmental regulation of proline transport in Leishmania donovani. Exp. Parasitol. 91:341-348.

46. Basselin, M., G.H. Coombs, and M.P. Barrett. 2000. Putrescine and spermidine transport in Leishmania. Mol. Biochem. Parasitol. 109:37-46.

47. Sacks, D., and A. Sher. 2002. Evasion of innate immunity by parasitic protozoa. Nat. Immunol. 3:1041-1047.
48. Spath, G.F., L.F. Lye, H. Segawa, S.J. Turco, and S.M. Beverley. 2004 Identification of a compensatory mutant (lpg2-REV) of Leishmania major able to survive as amastigotes within macrophages without LPG2-dependent glycoconjugates and its significance to virulence and immunization strategies. Infect. Immun. 72:3622-3627.

49. Mottram, J.C., G.H. Coombs, and J. Alexander. 2004. Cysteine peptidases as virulence factors of Leishmania. Curr. Opin. Microbiol. 7:375-381.

50. Courret, N., E. Prina, E. Mougneau, E.M. Saraiva, D.L. Sacks, N. Glaichenhaus, and J.C. Antoine. 1999. Presentation of the Leishmani antigen LACK by infected macrophages is dependent upon the virulence of the phagocytosed parasites. Eur. J. Immunol. 29:762-773.

51. Mumberg, D., R. Muller, and M. Funk. 1995. Yeast vectors for the controlled expression of heterologous proteins in different genetic backgrounds. Gene. 156:119-122.

52. Roy, D., D.R. Liston, V.J. Idone, A. Di, D.J. Nelson, C. Pujol, J.B. Bliska, S. Chakrabarti, and N.W. Andrews. 2004. A process for controlling intracellular bacterial infections induced by membrane injury. Science. 304:1515-1518.

53. Blackwell, J.M., S. Toole, M. King, P. Dawda, T.I. Roach, and A Cooper. 1988. Analysis of Lsh gene expression in congenic B10.L-Lshr mice. Curr. Top. Microbiol. Immunol. 137:301-309.

54. Roach, T.I., D. Chatterjee, and J.M. Blackwell. 1994. Induction of early-response genes $\mathrm{KC}$ and JE by mycobacterial lipoarabinomannans: regulation of $\mathrm{KC}$ expression in murine macrophages by Lsh/Ity/Bcg (candidate Nramp). Infect. Immun. 62:1176-1184.

55. Spath, G.F., and S.M. Beverley. 2001. A lipophosphoglycan-independent method for isolation of infective Leishmania metacyclic promastigotes by density gradient centrifugation. Exp. Parasitol. 99:97-103.

56. Tabbara, K.S., N.C. Peters, F. Afrin, S. Mendez, S. Bertholet, Y. Belkaid, and D.L. Sacks. 2005. Conditions influencing the efficacy of vaccination with live organisms against Leishmania major infection. Infect. Immun. 73:4714-4722. 